
PRAMANA
- journal of
physics
(c) Indian Academy of Sciences

西
pp. $1-16$

\title{
Sheared Solid Materials
}

\author{
Akira Onuki, Akira Furukawa, and Akihiko Minami \\ Department of Physics, Kyoto University, Kyoto 606-8502, Japan
}

\begin{abstract}
We present a time-dependent Ginzburg-Landau model of nonlinear elasticity in solid materials. We assume that the elastic energy density is a periodic function of the shear and tetragonal strains owing to the underlying lattice structure. With this new ingredient, solving the equations yields formation of dislocation dipoles or slips. In plastic flow high-density dislocations emerge at large strains to accumulate and grow into shear bands where the strains are localized. In addition to the elastic displacement, we also introduce the local free volume $m$. For very small $m$ the defect structures are metastable and long-lived where the dislocations are pinned by the Peierls potential barrier. However, if the shear modulus decreases with increasing $m$, accumulation of $m$ around dislocation cores eventually breaks the Peierls potential leading to slow relaxations in the stress and the free energy (aging). As another application of our scheme, we also study dislocation formation in two-phase alloys (coherency loss) under shear strains, where dislocations glide preferentially in the softer regions and are trapped at the interfaces.
\end{abstract}

Keywords. plastic flow, dislocations,free volume,aging,two-phase alloys, incoherency

\section{Introduction}

Nonequilibrium states under shear have been extensively studied for near-critical fluids and for various complex fluids including polymers, liquid crystals, surfactant systems, colloidal suspensions and so on [1]. In the soft condensed matter physics, considerable attention has also been paid to jamming rheology observed in sheared states of supercooled liquids, soft glassy materials such as dense microemulsions or granular materials [2]. In these systems while the particle size ranges from microscopic to macroscopic lengths, universal constrained dynamics is realized under external shear forces. We mention that mesoscopic dynamic heterogeneity and strong shear-thinning behavior have been observed in supercooled liquids (at relatively high $T$ ) [3-5] and dense microemulsions (at effectively low $T$ ) [6]. In engineering, on the other hand, plastic flow has long been studied in crystalline and amorphous solids and in glassy polymers [7-10]. In crystals irreversible motions of dislocations give rise to plastic deformations and large strains produce high-density dislocations. In amorphous solids shear strains tend to be localized in narrow shear bands in plastic flow above a yield stress. The width of such shear bands is microscopic in the initial stage but can grow to mesoscopic sizes, sometimes resulting in fracture. Shear bands were numerically realized at large shear strains in molecular dynamics simulations of two-dimensional (2D) two-component glasses 
$[11,12]$ and in simulations of a 2D phenomenological model [13]. Shear bands were observed also in granular materials (at effectively zero temperature) [14].

We also stress relevance of dislocations in various phase transformations in solids. Since the first work by Cahn [15] most theoretical studies have been focused on the coherent case in which the lattice planes are continuous through the interfaces $[1,16,17]$. In the incoherent case dislocations appear around the interfaces and the continuity is lost partially or even completely. Such incoherent microstructures emerge in various alloys when the lattice constants or the crystalline structures of the two phases are not close $[18,19]$. Moreover, they are produced in plastic flow because dislocations generated tend to be trapped at the interfaces [20]. Mechanical properties of two-phase solids are hence very different from those of one-phase solids. Simulation studies in this area are still at the beginning [21-23].

In this paper we will present our recent nonlinear elasticity theory to understand these diverse effects in 2D [24-26]. Our approach is phenomenological, but its merit over microscopic molecular dynamics simulations is that we can put emphasis on any aspects of the phenomena by controlling the parameters or changing the model itself. In Section 2 we will present a first version of our dynamic model, where the gross variables are the elastic displacement $\boldsymbol{u}$ and its velocity $\boldsymbol{v}_{\mathrm{L}}=\partial \boldsymbol{u} / \partial t$. The stress-strain relation and emergence of plastic flow at large strains will be discussed. Metastability of the resultant defect structures will be ascribed to the Peierls potential $[8,27]$. In Section 3 we will introduce a free-volume field $m$ [28] into our dynamic model, which will drastically change the dynamics of dislocations on long time scales. As a result, we can gain insights into the physical mechanism of aging in amorphous solids [29-33]. We expect that $m$ is a key order parameter for amorphous solids or glasses. In Section 4 we will include the composition difference $\psi=c_{A}-c_{B}$ for binary alloys to numerically realize dislocation networks in twophase states under shear strain. Such complex effects have rarely been studied in physics but are of great technological importance.

\section{Mechanical model}

\subsection{Nonlinear elastic energy}

We present a nonlinear elasticity model in $2 \mathrm{D}[24,25]$. In terms of the displacement $\boldsymbol{u}=\left(u_{x}, u_{y}\right)$ from a reference crystal state, we define the strain components as

$$
\begin{aligned}
& e_{1}=\nabla_{x} u_{x}+\nabla_{y} u_{y}, \\
& e_{2}=\nabla_{x} u_{x}-\nabla_{y} u_{y}, \\
& e_{3}=\nabla_{x} u_{y}+\nabla_{y} u_{x},
\end{aligned}
$$

where $\nabla_{x}=\partial / \partial x$ and $\nabla_{y}=\partial / \partial y$. We call $e_{1}$ the dilation strain, $e_{2}$ the tetragonal strain, and $e_{3}$ the shear strain. If we suppose a $2 \mathrm{D}$ triangular (or square) lattice, the elastic energy should be invariant with respect to the rotations of the reference frame by $\pm n \pi / 3$ (or $\pm n \pi / 2)(n=1,2, \cdots)$. Under rotation of the reference frame by $\theta$, the shear strains $e_{2}$ and $e_{3}$ are changed to

$$
e_{2}^{\prime}=e_{2} \cos 2 \theta+e_{3} \sin 2 \theta, \quad e_{3}^{\prime}=e_{3} \cos 2 \theta-e_{2} \sin 2 \theta .
$$



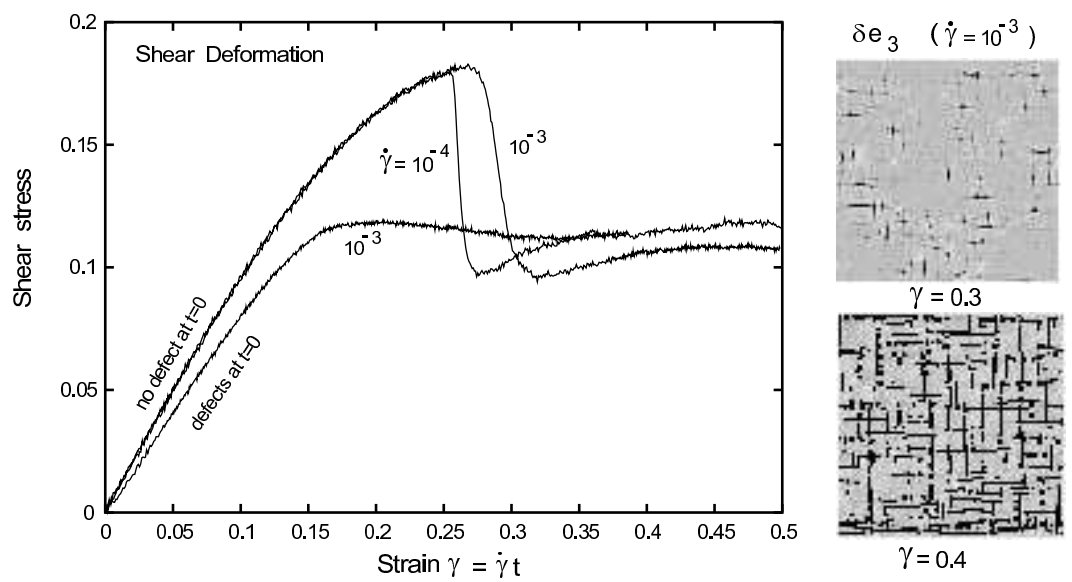

Figure 1. Stress-strain curves from (2.6). Those at $\dot{\gamma}=10^{-4}$ and $10^{-3}$ without defects at $t=0$ exhibit pronounced overshoot. Snapshots of $\delta e_{3}=e_{3}-\gamma$ are given at $\gamma=0.3$ and 0.4 (right). No marked overshoot appears in the other curve at $\dot{\gamma}=10^{-3}$, where high-density dislocations are initially present.

The elastic energy is written as $F=\int d \boldsymbol{r} f_{\text {el }}$ with the density in the form,

$$
f_{\mathrm{el}}=\frac{1}{2} K e_{1}^{2}+\Phi\left(e_{2}, e_{3}\right),
$$

where $K$ is the bulk modulus assumed to be a constant and $\Phi$ is the shear deformation energy. For a triangular lattice the simplest form of $\Phi$ is given by

$$
\Phi=\frac{\mu}{6 \pi^{2}}\left[3-\cos \pi\left(\sqrt{3} e_{3}-e_{2}\right)-\cos \pi\left(\sqrt{3} e_{3}+e_{2}\right)-\cos \left(2 \pi e_{2}\right)\right],
$$

which is invariant with respect to the rotation by $\pi / 6$ in (2.2), is a periodic function of $e_{3}$ with period $2 / \sqrt{3}$ for $e_{2}=0$ (simple shear deformation), and becomes the usual form $\mu\left(e_{2}^{2}+e_{3}^{2}\right) / 2$ in linear elasticity for small strains. A characteristic feature is that $\Phi$ in (2.4) is highly isotropic around its minima in the $e_{2}-e_{3}$ plane. That is, around the reference state $e_{2}=e_{3}=0, \Phi$ may be teated as a function of $e=\left(e_{2}^{2}+e_{3}^{2}\right)^{1 / 2}$ only for $e \lesssim 1 / 2$. For a square lattice structure we have recently used $[26]$

$$
\Phi=\frac{\mu_{2}}{4 \pi^{2}}\left[1-\cos \left(2 \pi e_{2}\right)\right]+\frac{\mu_{3}}{4 \pi^{2}}\left[1-\cos \left(2 \pi e_{3}\right)\right],
$$

which becomes $\left(\mu_{2} e_{2}^{2}+\mu_{3} e_{3}^{2}\right) / 2$ for small strains. One of the principal crystal axes is parallel to the $x$ axis in (2.4), while it is along or make an angle of $\pi / 4$ with respect to the $x$ axis in (2.5). If we allow the system to rotate as a whole, $f_{\mathrm{el}}$ becomes dependent on the rotational strain $\omega=\nabla_{x} u_{y}-\nabla_{y} u_{x}$. In this sense our model is still incomplete and further generalization is needed, particularly in describing polycrystalline states or melting phenomena. 


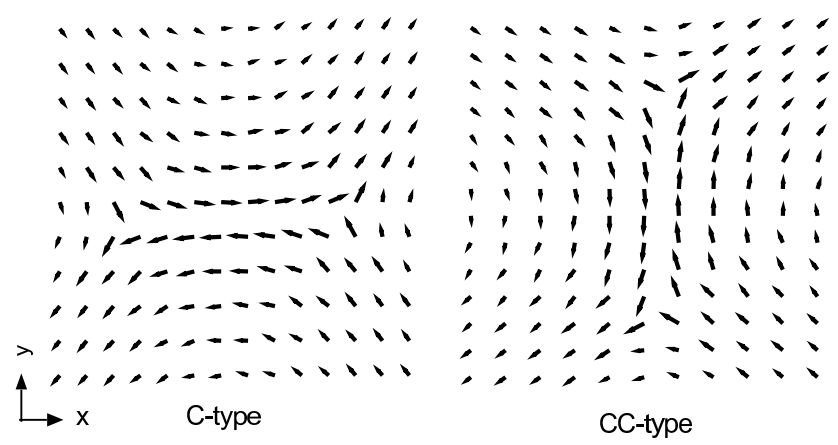

Figure 2. Displacement $\boldsymbol{u}$ around type $\mathrm{C}$ and type CC slips in the most favorable orientation in shear strain. The arrows are from the original undeformed position to the displaced position.

\subsection{Dynamics}

We assume that the lattice velocity $\boldsymbol{v}_{\mathrm{L}}=\partial \boldsymbol{u} / \partial t$ obeys

$$
\rho \frac{\partial}{\partial t} \boldsymbol{v}_{\mathrm{L}}=\nabla \cdot \stackrel{\leftrightarrow}{\sigma}++\eta_{0} \nabla^{2} \boldsymbol{v}_{\mathrm{L}}+\nabla \cdot \stackrel{\leftrightarrow}{\sigma}_{\mathrm{R}}
$$

where the stress tensor $\stackrel{\leftrightarrow}{\sigma}=\left\{\sigma_{i j}\right\}$ is given by

$$
\sigma_{x x}=K e_{1}+\frac{\partial \Phi}{\partial e_{2}}, \quad \sigma_{y y}=K e_{1}-\frac{\partial \Phi}{\partial e_{2}}, \quad \sigma_{x y}=\sigma_{y x}=\frac{\partial \Phi}{\partial e_{3}} .
$$

It follows the relation $\nabla \cdot \stackrel{\leftrightarrow}{\sigma}=-\delta F / \delta \boldsymbol{u}$. The mechanical equilibrium condition $\nabla \cdot \stackrel{\leftrightarrow}{\sigma}=\mathbf{0}$ is equivalent to the extremum condition $\delta F / \delta \boldsymbol{u}=\mathbf{0}$. We introduce the viscosity $\eta_{0}$ and the random stress tensor $\stackrel{\leftrightarrow}{\sigma}_{\mathrm{R}}$ [34]. We integrated (2.6) in $2 \mathrm{D}$ on a $128 \times 128$ square lattice with the mesh size equal to the lattice constant $a$. Use is made of (2.4) (where a triangular lattice is supposed), but almost the same results follow also using $\Phi$ in (2.5). Here $\mu$ is assumed to be a constant $\mu_{0}$. Space and time are measured in units of $a$ and $\tau_{0}=\left(\rho / \mu_{0}\right)^{1 / 2} a$. The dimensionless viscosity is $\eta_{0}^{*}=\eta_{0} / \tau_{0} \mu_{0}$ and is set equal to 1 . Spatial derivatives are appropriately defined to yield well-defined microscopic slips.

Fig.1 displays the stress-strain curves at constant shear rate $\dot{\gamma}$ applied for $t>0$ in units of $\mu_{0}$ and $\tau^{-1}$. For the curves of $\dot{\gamma}=10^{-3}$ and $10^{-4}$ with the pronounced peak, we set $\boldsymbol{u}=\mathbf{0}$ at $t=0$ supposing a perfect crystal at $t=0$. They approach the elastic instability point $\gamma=\sqrt{3} / 6$, where $\partial \sigma_{x y} / \partial \gamma=0$. Then the shear stress drops sharply with catastrophic slip formation. See the two snapshots of $\delta e_{3}=e_{3}-\gamma$. For the other curve at $\dot{\gamma}=10^{-3}$ we initially put high-density dislocations produced after cyclic shearing [25]. The stress overshoot is suppressed with increasing the initial disorder. In addition, the slip spacing depends on $\dot{\gamma}$ in plastic flow. For $\dot{\gamma}=10^{-4}$ the spacing is a few times wider than in the case of $\dot{\gamma}=10^{-3}$. 


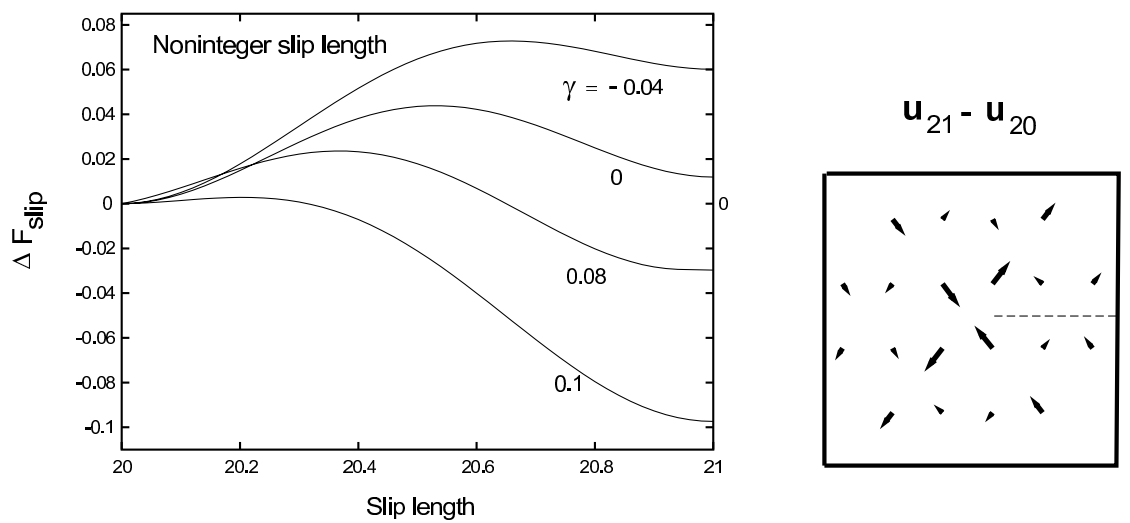

Figure 3. Slip energy difference $\Delta F_{\text {slip }}(\ell)$ in the range $20 \leq \ell \leq 21$ for $\gamma=-0.04,0,0.08$, and 0.1 in the model without free volume (left). Displacement difference $\boldsymbol{u}_{21}-\boldsymbol{u}_{20}$ at $\gamma=0$ needed for growth by unit length (right).

\subsection{Slips and Peierls potential}

A fundamental flow unit in our problem is a slip composed of a pair of edge dislocations. As shown in Fig.2, slips in 2D are divided into those of clockwise (C) type and those of counterclockwise (CC) type. A slip along one of the crystal axes is metastable without applied strains, where the slip length $\ell a$ is an integer multiple of the lattice constant $a$. It satisfies the mechanical equilibrium condition and can be calculated numerically. For noninteger $\ell$ there arises an increase of the elastic energy, $U_{\mathrm{P}}(\ell)$, called the Peierls potential [27]. Furthermore, under applied strains, $\left\langle e_{3}\right\rangle=\gamma$ and $\left\langle e_{2}\right\rangle=\epsilon$, the free energy to create a slip is given by [25]

$$
F_{\text {slip }}=\frac{\ln \ell}{2 \pi(1-\nu)} \mp(\gamma \cos 2 \varphi-\epsilon \sin 2 \varphi) \ell+U_{\mathrm{P}}(\ell),
$$

in units of $\mu a^{2}$ for the nearly isotropic $\Phi$ in (2.4). Here $\nu=(1-\mu / K) / 2, \varphi$ is the angle of the slip with respect to the $x$ axis, and $U_{\mathrm{P}}(\ell)$ vanishes for integer $\ell$. In front of the second term, - is for type $\mathrm{C}$ and + is for type CC. For simple shear deformation with $\gamma>0$ and $\epsilon=0$, the most favorable slip orientation with the lowest $F_{\text {slip }}$ is $\varphi=0$ for type $\mathrm{C}$ and $\varphi=\pi / 2$ for type CC as in Fig.2. For uniaxial stretching with $\gamma=0$ and $\epsilon>0$, it is given by $\varphi=-\pi / 4$ for type C and $\varphi=\pi / 4$ for type CC. In agreement with the last relations shear bands under uniaxial deformation have been observed to make angles of $\pm \pi / 4$ with respect to the stretched direction in isotropic amorphous solids [10-13] and granular materials [14]. In real crystals slip orientations can be strongly influenced by crystal anisotropy.

For noninteger $\ell, U_{\mathrm{P}}(\ell)$ is path-dependent. Here we calculate $F_{\text {slip }}(\ell)$ in the range $20 \leq \ell \leq 21$ for a type $\mathrm{C}$ slip parallel to the $x$ axis under shear strain $\gamma$. Use is made of the linearly extrapolated displacement,

$$
\boldsymbol{u}_{\ell}=(1-\alpha) \boldsymbol{u}_{20}+\alpha \boldsymbol{u}_{21}
$$

where $\alpha=\ell-20$. The $\boldsymbol{u}_{20}$ and $\boldsymbol{u}_{21}$ are numerically obtained for $\ell=20$ and 21 . Fig. 3 shows the free energy difference (left) 

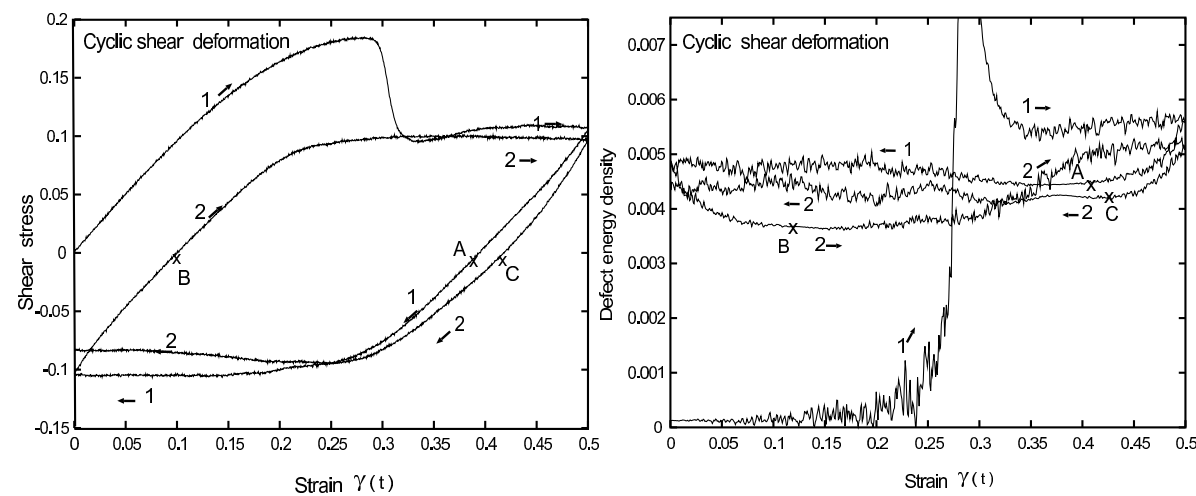

Figure 4. Shear stress $\left\langle\sigma_{x y}\right\rangle$ (left) and defect energy density $f_{\mathrm{D}}$ defined by (2.12) (right) vs strain $\gamma(t)$ for cyclic shear deformation at $\dot{\gamma}= \pm 10^{-3}$. The shear stress vanishes at the three points $\mathrm{A}, \mathrm{B}$, and $\mathrm{C}(\times)$.

$$
\Delta F_{\text {slip }}=F_{\text {slip }}(\ell)-F_{\text {slip }}(20),
$$

which exhibits a maximum at an intermediate $\ell$. The maximum approaches 20 as $\gamma \uparrow \gamma_{\mathrm{c} 1}$ and 21 as $\gamma \downarrow \gamma_{\mathrm{c} 2}$, where $\gamma_{\mathrm{c} 1} \sim 0.1$ and $\gamma_{\mathrm{c} 2} \sim-0.1$ here. Thus the slip is metastable for $\gamma_{\mathrm{c} 2}<\gamma<\gamma_{\mathrm{c} 1}$, but it expands for $\gamma>\gamma_{\mathrm{c} 1}$ and shrinks for $\gamma<\gamma_{\mathrm{c} 2}$. Fig. 3 also indicates that about 10 particles move significantly when the right end of the slip moves by unit length (right). Against thermal agitations slips can be long-lived if the maximum of $\Delta F_{\text {slip }}$ is much larger than $k_{\mathrm{B}} T$.

\subsection{Cyclic shear and defect energy density}

Next we apply a cyclic shear deformation, where $\dot{\gamma}(t)=10^{-3}$ in the time regions $n t_{\mathrm{p}}<t<(n+1 / 2) t_{\mathrm{p}}$ and $\dot{\gamma}(t)=-10^{-3}$ in the time regions $(n+1 / 2) t_{\mathrm{p}}<t<$ $(n+1) t_{\mathrm{p}}$. For the first two cycles, Fig. 4 shows the stress-strain curve (left) and a defect energy density $f_{\mathrm{D}}$ (right). The latter is defined as follows. We divide the strain $\gamma(t)$ into an elastic strain $\gamma_{\mathrm{el}}$ and a slip strain $\gamma_{\mathrm{s}}=\gamma-\gamma_{\mathrm{el}}$. Roughly speaking, the elastic strain outside the slip lines gives rise to the average stress, while the slip strain is caused by the jumps of $u_{x}$ across the slips. Regarding the shear stress in (2.7) as a function of $e_{2}$ and $e_{3}$, we define $\gamma_{\mathrm{el}}$ by

$$
\sigma_{x y}\left(0, \gamma_{\mathrm{el}}\right)=\left\langle\sigma_{x y}\right\rangle .
$$

We define the average defect energy density by

$$
f_{\mathrm{D}}=\left\langle f_{\mathrm{el}}\right\rangle-\Phi\left(0, \gamma_{\mathrm{el}}\right),
$$

where $\Phi\left(e_{2}, e_{3}\right)$ is given by (2.4). The elastic energy density stored is then the sum of the regular elastic energy density and the defect energy density. For small $\gamma_{\mathrm{el}}$ 
we have $\gamma_{\mathrm{el}} \cong\left\langle\sigma_{x y}\right\rangle / \mu_{0}$ and $\Phi \cong \mu_{0} \gamma_{\mathrm{el}}^{2} / 2$. In Fig.4 we notice (i) residual strains at vanishing stress at points $\mathrm{A}, \mathrm{B}$, and $\mathrm{C}$, (ii) no overshoot in the stress from the second cycle, (iii) that $\left\langle\sigma_{x y}\right\rangle<0$ at the ends of each cycle, and (iv) that $\left\langle\sigma_{x y}\right\rangle(t)$ and $f_{\mathrm{D}}(t)$ are roughly constant in plastic flow.

For $\gamma(t) \lesssim 0.3$ in the first cycle, however, $f_{\mathrm{D}}(t)$ represents the elastic energy due to the inhomogeneous fluctuations of the local strains (mainly due to $\delta e_{3}$ ) with the peak height at 0.012 (not shown in the figure). After this initial period, $f_{\mathrm{D}}(t)$ is in a range of $0.004-0.005$ reasonably representing the elastic energy due to the defects produced in plastic flow. Note that $f_{\mathrm{D}}(t)$ depends on $\dot{\gamma}$ and it is almost constant around 0.002 in plastic flow with $\dot{\gamma}= \pm 10^{-4}$.

\section{Dynamic free-volume model}

\subsection{Free volume}

In the presence of point defects or local free volume, there can be a difference between the normalized density deviation $\delta \rho / \rho$ and $-e_{1}$ even in the linear elasticity. Cohen, Flemming, and Gibbs [35] constructed a hydrodynamic description of solids including a new variable which they called the vacancy field. Its small deviation $\left(=\delta \rho / \rho+e_{1}\right)$ was predicted to relax diffusively (vacancy diffusion). More generally, since vacancies are point defects, let $m$ represent the local free-volume fraction defined at each lattice point [28], which can take continuous values. Here we introduce $m$ in our nonlinear elasticity scheme [24].

As a reference state we suppose a closely packed crystal state with $v_{0}$ being a unit cell volume (or area in 2D). We consider the cell number density $n_{\text {cell }}$, which changes with lattice dilation, and we define $m$ as

$$
m=v_{0}\left(n_{\text {cell }}-\rho / M\right),
$$

where $M$ is the mass in a unit volume of the reference state (=constant). We assume that $m$ is non-negative $(m \geq 0)$ neglecting excess packing such as interstitials. If

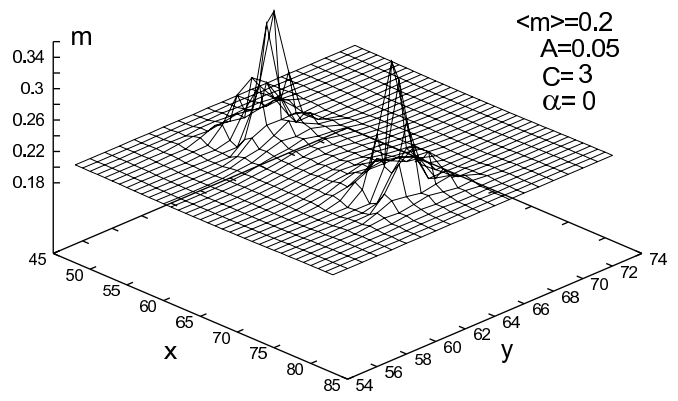

Figure 5. Free volume $m$ accumulated at the dislocation cores due to $m$-dependence of the shear modulus. 

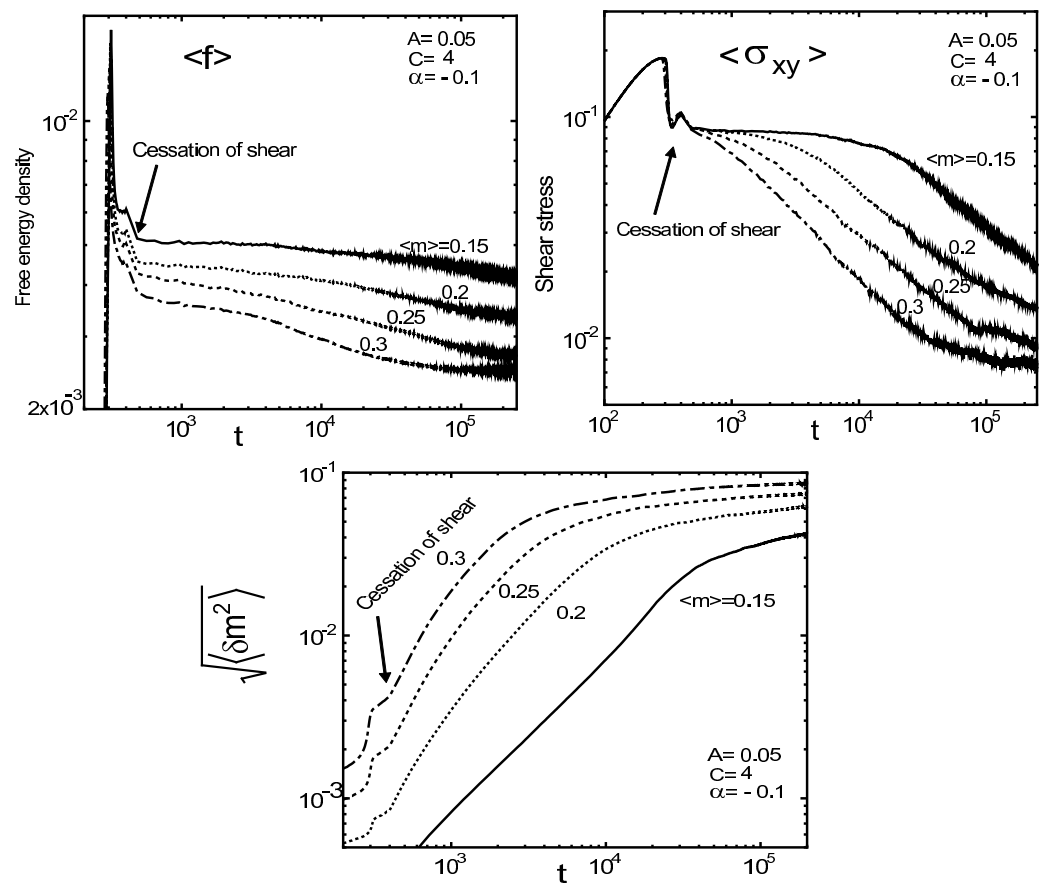

Figure 6. Free energy density $\langle f\rangle(t)$, shear stress $\left\langle\sigma_{x y}\right\rangle(t)$, and free-volume variance $\sqrt{\left\langle\delta m^{2}\right\rangle}(t)$ vs $t$. A shear flow with $\dot{\gamma}=10^{-3}$ is applied in the time region $0<t<400$ and is stopped at $t=400$. Relaxations become apparent with increasing the average free volume $\langle m\rangle$.

the density deviation $\delta \rho$ is small compared with the average density $\bar{\rho}, m$ may be approximated as

$$
m=\langle m\rangle-\left(e_{1}+\delta \rho / \bar{\rho}\right) .
$$

The average free volume $\langle m\rangle=v_{0}\left(\left\langle n_{\text {cell }}\right\rangle-\bar{\rho} / M\right)$ decreases with lowering $T$ and/or increasing $\bar{\rho}$ and represents the distance of the system from the closely packed perfect crystal. If the total volume of the system is fixed, $m$ is a conserved variable and $\langle m\rangle$ is a constant independent of time. We shall see that $\langle m\rangle$ is an important parameter controlling slow relaxations of the defect structures.

\subsection{Free energy and dynamic equations}

In this work we use the following free energy density,

$$
f=A m \ln (m /\langle m\rangle)+\alpha m e_{1}+\frac{1}{2} K e_{1}^{2}+\Phi\left(m, e_{2}, e_{3}\right) .
$$



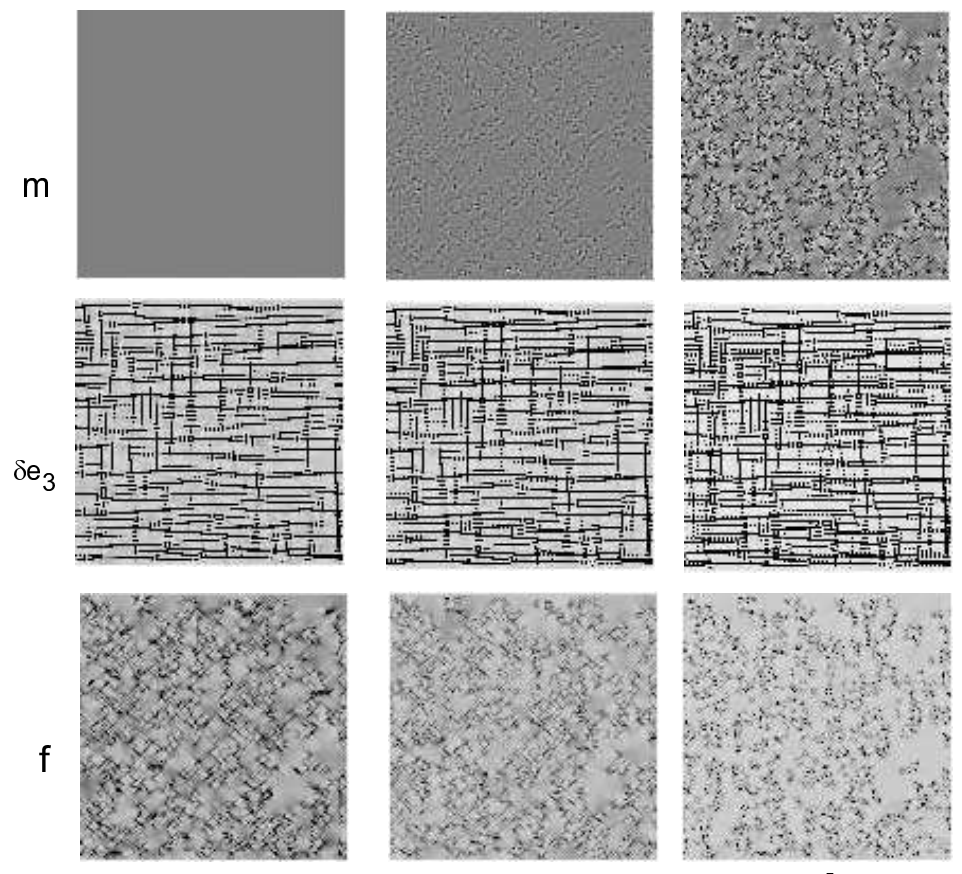

$t=400$

$\mathrm{t}=3200$

$$
t=2 \times 10^{5}
$$

Figure 7. Snapshots of free volume $m$, shear strain deviation $\delta e_{3}$, and free energy density $f$ at $t=400,3200$, and $2 \times 10^{5}$ for $\langle m\rangle=0.2$, showing growth of heterogeneity of $m$, an increase of plastic strain, and a decrease of $f$.

The first term is an entropic term, though its form is not well justified. However, we may well replace it by the simpler form $A^{\prime} m^{2} / 2$ without loss of essential results of our simulations. The coupling parameter $\alpha$ is assumed to be small since $|\delta m| \ll$ $\left|\delta e_{1}\right|$ should hold except close to the dislocation cores. We use the nearly isotropic $\Phi$ in (2.4), but the shear modulus $\mu$ decreases exponentially with increasing $m$ as

$$
\mu(m)=\mu_{0} \exp (-C \delta m),
$$

where $\delta m=m-\langle m\rangle$ and $C$ is a positive constant. This relation simply arises from the requirement that solids should soften with increasing the free volume. The same exponential form was used to describe softening due to point defects [36]. If $|\delta \rho| \ll \bar{\rho}$ and the strains are small, the mass density deviation $\delta \rho$ obeys

$$
\frac{\partial}{\partial t} \delta \rho=-\bar{\rho} \nabla \cdot \boldsymbol{v}
$$

The mass velocity $\boldsymbol{v}$ is governed by

$$
\bar{\rho} \frac{\partial}{\partial t} \boldsymbol{v}=\nabla \cdot \stackrel{\leftrightarrow}{\sigma}+\eta_{0} \nabla^{2} \boldsymbol{v}+\nabla \cdot \stackrel{\leftrightarrow}{\sigma}_{\mathrm{R}}
$$


Including the contribution from the pressure deviation $\bar{\rho}(\delta F / \delta \rho) \boldsymbol{u}$ the force density on the right hand side is written in terms of $F=\int d \boldsymbol{r} f$ as

$$
\nabla \cdot \stackrel{\leftrightarrow}{\sigma}=-\bar{\rho} \nabla\left(\frac{\delta F}{\delta \rho}\right)_{\boldsymbol{u}}-\left(\frac{\delta F}{\delta \boldsymbol{u}}\right)_{\rho}=-\left(\frac{\delta F}{\delta \boldsymbol{u}}\right)_{m}
$$

where use has been made of the identities $(\delta / \delta \boldsymbol{u})_{\rho}=(\delta / \delta \boldsymbol{u})_{m}-\nabla(\delta / \delta m) \boldsymbol{u}$ and $(\delta / \delta m) \boldsymbol{u}=-\bar{\rho}(\delta / \delta \rho) \boldsymbol{u}$. The expressions for $\sigma_{i j}$ follow from (2.7) if $K e_{1}$ is replaced by $K e_{1}+\alpha m$ and the derivatives are taken at fixed $m$. The lattice velocity $\partial \boldsymbol{u} / \partial t$ is different from the mass velocity $\boldsymbol{v}$ and is of the form [35],

$$
\frac{\partial}{\partial t} \boldsymbol{u}=\boldsymbol{v}_{\mathrm{L}}=\boldsymbol{v}-L(m)\left(\frac{\delta F}{\delta \boldsymbol{u}}\right)_{\rho}+\boldsymbol{\zeta}_{\mathrm{R}}
$$

where $L(m)$ is the kinetic coefficient dependent on $m$ and $\boldsymbol{\zeta}_{\mathrm{R}}$ is the random velocity related to $L(m)$ via the fluctuation-dissipation theorem. These dynamic equations are approximate. However, if the random forces are neglected, the total free energy $F_{T}=\int d \boldsymbol{r}\left[f+\bar{\rho} \boldsymbol{v}^{2} / 2\right]$ decreases in time without external forces. The system becomes steady only in mechanical equilibrium $\nabla \cdot \stackrel{\leftrightarrow}{\sigma}=\mathbf{0}$.

From (3.5)-(3.8) we derive the equation for $m$,

$$
\frac{\partial}{\partial t} m=\nabla \cdot L(m)\left[\nabla\left(\frac{\delta F}{\delta m}\right)_{\boldsymbol{u}}-\nabla \cdot \stackrel{\leftrightarrow}{\sigma}\right]+\nabla \cdot \boldsymbol{\zeta}_{\mathrm{R}}
$$

If the couplings to $\boldsymbol{u}$ are neglected, it follows the nonlinear diffusion equation $\partial m / \partial t=\nabla \cdot D(m) \nabla m$ with $D(m)=L(m) A / m$. Because the diffusion of $m$ should be drastically slowed down for small $m$, we assume the following form,

$$
D(m)=D_{0} \exp (-B / m),
$$

where $D_{0}$ and $B$ are positive constants. This form is also not well justified. It is worth noting that the same form was used for the relaxation rate of the free volume in granular matters [37]. Another aspect is the presence of $\nabla \cdot \stackrel{\leftrightarrow}{\sigma}$ in the equation of $m$. A similar stress-diffusion coupling is well-known in the dynamic equation of the composition for gels and viscoelastic fluid mixtures $[1,38,39]$. See the last section for more comments.

\subsection{Slow relaxation due to free-volume accumulation}

We choose $\boldsymbol{v}, \boldsymbol{u}$, and $m$ as fundamental variables and solve (3.6),(3.8), and (3.9) by setting $A=0.05 \mu_{0}, B=1$, and $D_{0}=2 \times 10^{-3}$ [40]. In Fig.5 we first show a steady-state profile of $m$ around a slip for $\langle m\rangle=0.2, C=3$, and $\alpha=0$. Here the steady-state condition $\delta F / \delta m=$ const. yields

$$
m \propto \exp \left[\left(C \Phi-\alpha e_{1}\right) / A\right] .
$$

Accumulation of $m$ around the dislocation cores becomes signficant for small $A$ and large $C$. Such heterogeneity of $m$ should cause slow structural relaxations or aging, 

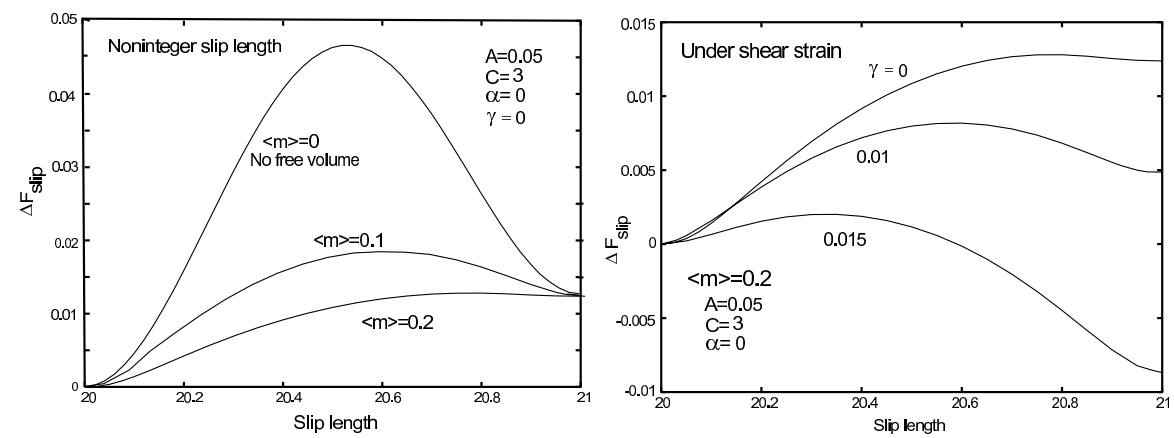

Figure 8. Slip energy difference $\Delta F_{\text {slip }}(\ell)$ in the range $20 \leq \ell \leq 21$ at $\gamma=0$ for $\langle m\rangle=0,0.1$, and 0.2 (right) and at $\langle m\rangle=0.2$ for $\gamma=0,0.01$, and 0.015 (left). The quasi-static condition $\delta F / \delta m=$ const.is assumed along the path connecting the steady slip solutions with $\ell=20$ and 21 .

as expected in the literature [29]. As an example, a shear flow at rate $\dot{\gamma}=10^{-3}$ is applied in the time region $0<t<400$ and is stopped at $t=400$. Fig.6 displays time-evolution of the average free energy density $\langle f\rangle(t)$, the average stress $\left\langle\sigma_{x y}\right\rangle(t)$, and the variance $\sqrt{\left\langle\delta m^{2}\right\rangle}(t)$. The last quantity represents heterogeneity of $m$. The four curves are those for $\langle m\rangle=0.15,0.2,0.25$, and 0.3 . The other parameter values are given in the figure. The relaxations are more apparent with increasing $\langle m\rangle$. We are adding very small Langevin noises [34], so noisy behavior appears only at very long times. Therefore, the relaxations here are due to deterministic diffusive accumulation of $m$ around randomly and densely distributed dislocations. If the noise amplitude is increased, the relaxations become faster and noisy.

The origin of the slow relaxations may be ascribed to weakening of the Peierls barrier with accumulation of $m$ at the dislocation cores. In Fig. 8 the slip energy $\Delta F_{\text {slip }}$ defined in (2.10) is calculated in the range $20 \leq \ell \leq 21$, where $\boldsymbol{u}$ is fixed as in (2.9) but $m$ is relaxed until $\nabla \delta F / \delta m$ nearly vanishes at each $\alpha$. Namely, $F$ is minimized with respect to $m$ at fixed $\boldsymbol{u}$. The slip-length change supposed here is quasi-static taking place only on time scales longer than $a^{2} / D(m)$. From Figs.3 and 8 we recognize that the maxima of $\Delta F_{\text {slip }}$ much decrease with increasing $\langle m\rangle$ and that the metastability is broken by applied strains much smaller than in the absence of free volume.

\section{Two-phase alloys}

\subsection{Composition coupled to nonlinear elasticity}

We consider a binary alloy composed of $A$ and $B$ atoms neglecting point defects. The compositions, $c_{A}$ and $c_{B}$, of the two components satisfy $c_{A}+c_{B}=1$. In real metallic alloys an order-disorder phase transition can also occur together with phase separation $[1,16,21]$. However, the composition difference $\psi=c_{A}-c_{B}$ is the sole order parameter in this work. The free energy density $f$ is of the form, 


$$
f=f_{\mathrm{BW}}(\psi)+\frac{C}{2}|\nabla \psi|^{2}+\alpha e_{1} \psi+\frac{K}{2} e_{1}^{2}+\Phi\left(\psi, e_{2}, e_{3}\right) .
$$

The first term is the Bragg-Williams free energy density [1],

$$
f_{\mathrm{BW}}=\frac{k_{\mathrm{B}} T}{v_{0}}\left[\frac{1+\psi}{2} \ln (1+\psi)+\frac{1-\psi}{2} \ln (1-\psi)\right]-\frac{k_{\mathrm{B}} T_{0}}{2 v_{0}} \psi^{2},
$$

where $v_{0}$ is the volume of a unit cell, $T_{0}$ is the mean-field critical temperature without coupling to the elastic field. For deep quenching this form is more appropriate than the Landau expansion form. The second term in (4.1) is the gradient free energy. There are two couplings between $\psi$ and the elastic field in $f$. First, $\alpha$ is the strength of the coupling to $e_{1}$ arising from a difference in the atomic sizes of the two species, leading to lattice misfit in two-phase states. In particular, around a dislocation it gives rise to a compositional Cottrell atmosphere [7] in one-phase states and preferential nucleation [42] in the metastable region. Second, the shear deformation energy $\Phi$ is of the form of (2.5) with

$$
\mu_{k}=\mu_{k 0}+\mu_{k 1} \psi \quad(k=2,3),
$$

where $\mu_{k 0}$ and $\mu_{k 1}$ are constants. If $\mu_{21}>0$ and $\mu_{31}>0$, regions with larger (smaller) $\psi$ are harder (softer) than those with smaller (larger) $\psi$. The inhomogeneity in the shear moduli, called elastic inhomogeneity, plays a decisive role in the domain morphology in late stages, because it gives rise to asymmetric elastic deformations in the two phases $[1,43,44]$.

In the coherent case the mechanical equilibrium condition $\nabla \cdot \stackrel{\leftrightarrow}{\sigma}=\mathbf{0}$ may be assumed even in dynamics. Here the stress tensor $\stackrel{\leftrightarrow}{\sigma}=\left\{\sigma_{i j}\right\}$ is expressed as in (2.7). Using this condition in the linear elasticity, the elastic field has been expressed in terms of $\psi$ in the previous theories [1,15-17]. We then find the following:

(i) The typical strain produced by the lattice misfit is given by

$$
e_{0}=\alpha \Delta \psi / 2 L_{0},
$$

where $\Delta \psi$ is the difference of $\psi$ between the two phases and $L_{0}\left(=K+\mu_{20}\right.$ in 2D) is the longitudinal modulus. In the coherent condition we require $e_{0}+\gamma \lesssim 1 / 4$ under applied strain $\gamma$. (ii) In the weak limit of cubic elasticity and elastic inhomogeneity, one-phase states become linearly unstable for $T<T_{\mathrm{s}} /\left(1+\langle\psi\rangle^{2} / 2\right)$, where

$$
T_{\mathrm{s}}=T_{0}+v_{0} \alpha^{2} / L_{0} k_{\mathrm{B}}
$$

is the spinodal temperature of one-phase states at the critical composition.

In the incoherent case the mechanical equilibrium does not hold when dislocations are created and are moving. We thus set up the dynamic equation (3.6) for $\boldsymbol{u}$. Then the damping of $\boldsymbol{u}$ is governed by the kinetic viscosity $\eta_{0} / \rho$. The composition obeys

$$
\frac{\partial \psi}{\partial t}=\nabla \cdot \lambda_{0}\left(1-\psi^{2}\right) \nabla \frac{\delta F}{\delta \psi}
$$

where $\boldsymbol{u}$ is fixed in $\delta F / \delta \psi$. We neglect Langevin noise terms in integrating these dynamic equations. In the dilute limit $c_{A} \rightarrow 0, c_{A}$ relaxes diffusively with the diffusion constant $D_{0}=\lambda_{0} k_{\mathrm{B}} T v_{0}^{-1}$. 

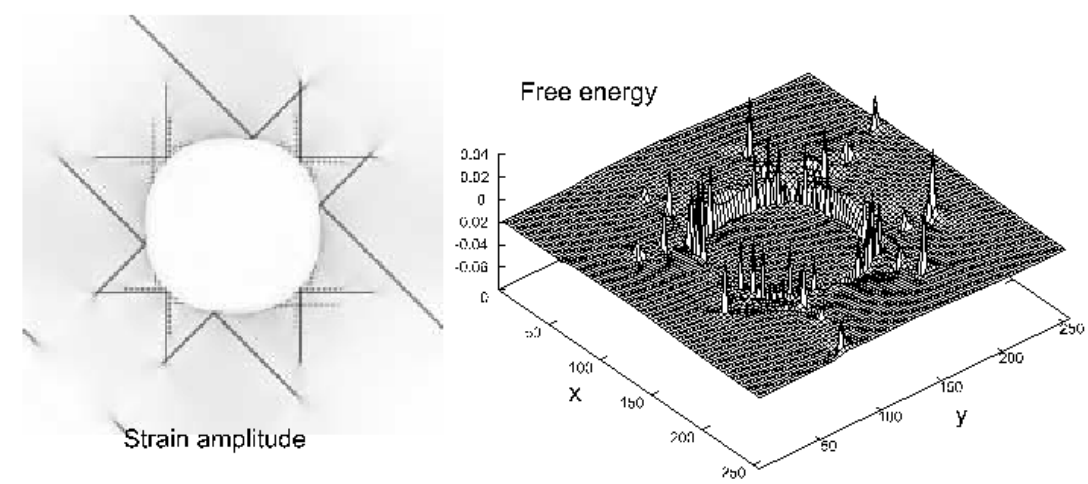

Figure 9. Strain amplitude $e$ (left) and free energy density $f$ (right) around an incoherent hard domain. Here $e$ is zero within the hard domain and nonvanishing outside. The peaks of $f$ are located at the dislocation cores.

\subsection{Dislocation formation}

We integrate the dynamic equations in $2 \mathrm{D}$ on a $256 \times 256$ square lattice under the periodic boundary condition by setting $K / \mu_{20}=4.5, \alpha / \mu_{20}=0.6, k_{\mathrm{B}} T_{0} / v_{0} \mu_{20}=$ $C / a^{2} \mu_{20}=0.05$, and $\mu_{21}=\mu_{31}=0.6 \mu_{20}$ [26]. Space and time are measured in units of $a$ and $\tau_{0}=\left(\rho / \mu_{20}\right)^{1 / 2} a$, respectively. We set the diffusion constant $D_{0}$ at $5 \times 10^{-5} \eta_{0} / \rho$ and the dimensionless viscosity $\eta_{0} / \tau_{0} \mu_{20}$ at 0.1 . The time scale of $\psi$ is much longer than that of the elastic field by four orders of magnitude. In real solid alloys, these two time scales are even more separated, probably except for hydrogen-metal systems where the protons diffuse quickly [1].

Fig.9 shows an incoherent hard domain obtained at deep quenching at $T / T_{0}=1$. Slight cubic anisotropy $\mu_{30} / \mu_{20}=1.1$ is assumed, so the domain shape is a rounded square. We displays the strain amplitude $e=\left(e_{2}^{2}+e_{3}^{2}\right)^{1 / 2}$ (left) and the free energy density $f$ in (4.1) (right). The slips make angles of $\pm \pi / 4$ with respect to the $x$ axis where $\left|e_{2}\right|$ is large, while they are parallel to the $x$ or $y$ axis in the corner regions with large $\left|e_{3}\right|$. In the interface region $f$ exhibits a cliff-like structure arising from

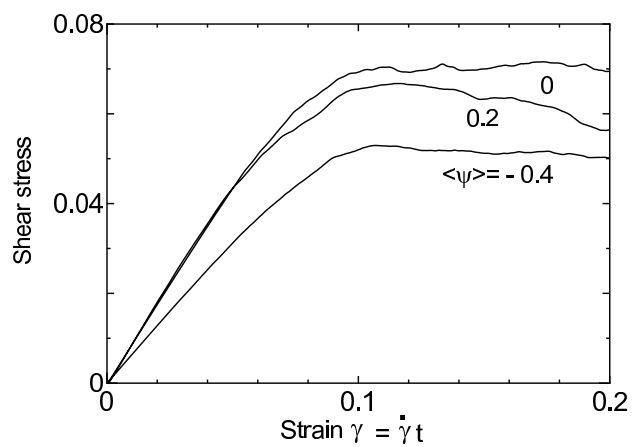

Figure 10. Stress-strain curves in two-phase states with $\langle\psi\rangle=-0.4,0$, and 0.2. There are no initial dislocations. Yield occurs with dislocation formation. 


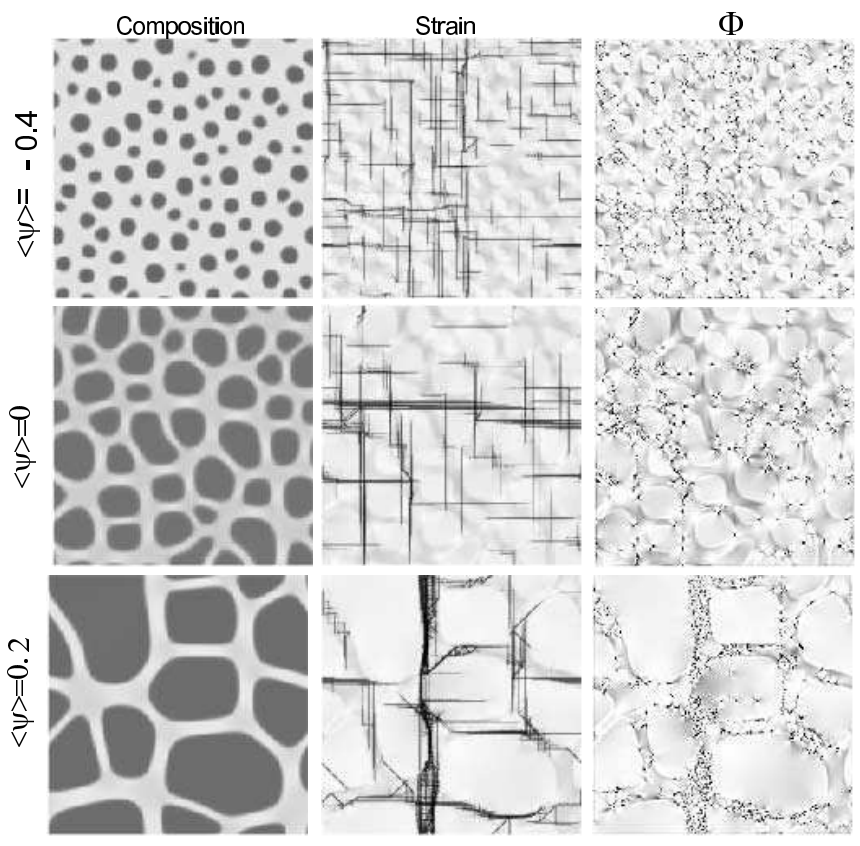

Figure 11. Composition difference $\psi$ (left), shear strain deviation $\delta e_{3}=e_{3}-\gamma$ (middle), and shear deformation energy $\Phi$ (right) in plastic flow at $\gamma=0.2$ in Fig.10. The average order parameter $\langle\psi\rangle$ is $-0.4,0$, and 0.2 from above. The black regions (right) represent harder domains, which are enclosed by the softer phase (in gray).

the gradient term and higher peaks arising from the dislocation cores. Here we remark that dislocation clouds have been observed around $\gamma^{\prime}$ precipitates of the $\mathrm{L} 1_{2}$ structure in alloys with large elastic misfit [19].

Next, in the isotropic condition $\mu_{20}=\mu_{30}$, we initially prepare coherent two-phase states at $T=2 T_{0}<T_{\mathrm{s}}$ for three average compositions. We then apply a shear flow at $\dot{\gamma}=10^{-3}$ for $t>0$. As can be seen in the stress-strain curves in Fig.10, yield occurs at $\gamma \sim 0.1$ where dislocations start to be created. In Fig.11 slips glide in the softer regions and stop at the interfaces. We can see that the black points in $\Phi$ representing dislocation cores are mostly trapped at the interfaces. These results are consistent with metallurgical experiments $[18,20]$.

\section{Summary and concluding remarks}

We have shown that the periodicity of the elastic energy density with respect to $e_{2}$ and $e_{3}$ gives rise to proliferation of dislocations under large strains. Furthermore, we have found new approaches to unexplored complex effects with introduction of a new variable, the free volume or the composition, coupled to the elastic field. We 
give some additional remarks.

(i) In our simulations slips emerge as long straight lines. If disorder is fully introduced, glide motions of slips in particular directions should be much limited. In molecular dynamics (MD) simulations of two component glasses, the degree of disorder should be sensitive to the size ratio of the two species. When it was rather

close to 1, long slips indeed emerged [11]. Performing MD simulations with various size ratios is thus informative.

(ii) We mention the effect of elastic interaction among dislocations. In our case slips are more easily created around preexisting ones, leading to growth of shear bands, as already reported in Ref. [13].

(iii) In Section3, (3.6), (3.8), and (3.9) constitute a two-fluid model. We expect that the fluctuations of $m$ and hence $\delta \rho$ can be increased anisotropically under applied strains and after migration of $m$ [24]. This effect is intensified for small $A$ in (3.3) and large $C$ in (3.4). In polymers and gels the composition fluctuations have been observed to increase under strains $[1,39]$.

(iv) In Section 4, the composition has been taken as a single order parameter. Extension is needed to more general phase ordering involving an order-disorder phase transition $[1,16,21]$ or diffusionless (Martensitic) structural phase transitions $[1,16]$.

(v) We conjecture that the origin of the dynamic heterogeneity observed in MD simulations [3-5] could be ascribed to long-range correlations of the free volume. This is because the structural relaxation is caused by the free-volume redistribution around elastic inhomogeneity.

(vi) Finally we stress that our nonlinear elasticity is still incomplete. For example, there should be the convective terms in (3.9) and (4.6). Also see the comment below (2.5). Construction of a more complete theory is under way.

\section{References}

[1] A. Onuki, Phase Transition Dynamics (Cambridge University, Cambridge, 2002).

[2] A. Liu and S.R. Nagel (eds.), Jamming and Rheology (Taylor \& Francis, London and New York, 2001). Here a number of related papers can be found.

[3] K. Maeda and S. Takeuchi, Phys. Status Solidi A 49, 685 (1978) ; S. Kobayashi, K. K. Maeda and S. Takeuchi, Acta Metall. 28, 1641 (1980).

[4] T. Muranaka and Y. Hiwatari, Phys. Rev. E 51, R2735 (1995); M.M. Hurley and P. Harrowell, Phys. Rev. E 52, 1694 (1995); W. Kob, C. Donati, S.J. Plimton, P.H. Poole, and S.C. Glotzer, Phys. Rev. Lett. 79, 2827 (1997).

[5] R. Yamamoto and A. Onuki, J. Phys. Soc. Jpn., 662545 (1997); Phys. Rev. E 58, 3515 (1998); J.-L. Barrat and L. Berthier, Phys. Rev. E 63, 012503 (2001).

[6] T. Okuzono and K. Kawasaki, Phys. Rev. E 51, 1246 (1995); D.J. Durian, Phys. Rev. E 55, 1739 (1997); S.A. Langer and A.J. Liu, J. Phys. Chem. B 101, 8667 (1997).

[7] A.H. Cottrell, Dislocations and Plastic Flow in Crystals (Clarendon, Oxford, 1953).

[8] F.R.N. Nabarro, Theory of Dislocations (Clarendon, Oxford, 1967).

[9] F. Spaepen, in Physics of Defects, edited by R. Balian, M. Kléman and J -P. Poirier, 1981 Les Houches Lectures (North-Holland, Amsterdam, 1981), p. 133.

[10] A.S. Argon, in Material sciences and technology, edited by R. W. Cohen, P. Haasen and E.J. Kramer (VCH, Weinheim, 1993), Vol. 6.

[11] D. Deng, A.S. Argon and S. Yip, Phil. Trans. R. Soc. Lond. A 329, 613 (1989).

[12] X.Y. Fu, D.A. Rigney and M.L. Falk, J. Non-Cryst. Solids 317, 206 (2003). 
[13] V. V. Bulatov, A.S. Argon, Modelling Simul. Mater. Sci. Eng. 2, 167, 185, 203 (1994).

[14] J. Desrues and R. Chambon, Int.J. Solids Struct. 39, 3757 (2002).

[15] J.W. Cahn, Acta Metall. 9, 795 (1961); 11, 1275 (1963).

[16] A.G. Khachaturyan, Theory of Structural Transformations in Solids (John Wiley \& Sons, New York, 1983).

[17] P. Fratzl, O. Penrose, and J.L. Lebowitz, J. Stat. Phys. 95, 1429 (1999).

[18] A.S. Argon, in Physical Metallurgy, edited by R.W. Cahn and P. Haasen (NorthHolland, Amsterdam, 1996), p.1878, 1958; P. Haasen, p.2010; J.L. Strudel, p.2106.

[19] E. A. Marquis and D. N. Seidman, Acta Mater. 48, 3477 (2000); M.J. Jones and F.J. Humphreys, ibid. 51, 2149 (2003); S.Iwamura and Y. Miura, ibid. 52, 591 (2004).

[20] T. M. Pollock and A. S. Argon, Acta Mater. 40, 1 (1992).

[21] F. Léonard and R. Desai, Phys. Rev. B, 58, 8277 (1988).

[22] S.Y. Hu, S.Schmauder, and L.Q. Chen, Phys. Status Solidi. B 220, 845 (2000); S.Y. $\mathrm{Hu}$ and L.Q. Chen, Acta Mater. 49, 463 (2001).

[23] Y. Wang, D. J. Srolovitz, J. M. Rickman and R. LeSar, Acta Mater., 48, 2163 (2000).

[24] A. Onuki, J. Phys. Condens. Matter 15, S891 (2003). Here we defined $m=e_{1}+\delta \rho / \bar{\rho}$.

[25] A. Onuki, Phys. Rev. E 68, 061502 (2003).

[26] A. Minami and A. Onuki, preprint.

[27] R. Peierls, Proc. Phys. Soc. 52, 34 (1940); F.R.N. Nabarro, ibid. 59, 256 (1947).

[28] D. Turnbull and M.H. Cohen, J. Chem. Phys. 523038 (1970).

[29] L.C.E. Struik, Physical Aging in Polymers and Other Amorphous Materials (Elsevier,Amsterdam, 1978).

[30] W. Kob, F. Sciortino and P. Tartaglia, Europhys. Lett. 49, 590 (2000).

[31] M. Utz, P.G. Debenedetti, and F.H. Stillinger, Phys. Rev. Lett. 84, 1471 (2000).

[32] D.J. Lacks, Phys. Rev. Lett. 87, 225502 (2001).

[33] L. Angelani, G. Ruocco, F. Sciortino, P. Tartaglia, and F. Zamponi, Phys. Rev. E 66, 061505 (2002).

[34] The dimensionless fluctuation-dissipation relation reads $\left\langle\sigma_{i j}^{\mathrm{R}}(\boldsymbol{r}, t) \sigma_{i j}^{\mathrm{R}}\left(\boldsymbol{r}^{\prime}, t^{\prime}\right)\right\rangle=$ $2 \epsilon_{\mathrm{th}} \eta_{0}^{*} \delta\left(\boldsymbol{r}-\boldsymbol{r}^{\prime}\right) \delta\left(t-t^{\prime}\right)$ with $\epsilon_{\mathrm{th}}=k_{\mathrm{B}} T / \mu_{0} a^{2}$. We set $\epsilon_{\mathrm{th}}=4 \times 10^{-5}$ in Fig.1 and $\epsilon_{\mathrm{th}}=10^{-5}$ in Figs. 6 and 7 . However, $\epsilon_{\mathrm{th}}=0$ for the other figures. For not very small Langevin noise the profiles of the dynamic variables become fuzzy and we need to perform many simulation runs and take statistical averages.

[35] P. D. Flemming III and C. Cohen, Phys. Rev. B 13, 500 (1976); C. Cohen, P.D. Flemming III, and J.H. Gibbs, Phys. Rev. B 13, 866 (1976).

[36] G.J. Dienes, Phys. Rev. 86, 228 (1952); F.R.N. Nabarro, Phys. Rev. 87, 665 (1952); A.V. Granato, J de Physique IV (C8) 6, 1 (1996).

[37] T. Boutreux and P. G. de Gennes, Physica A 224, 59 (1997).

[38] T. Tanaka, L.O. Hocker and G.B. Benedik, J. Chem. Phys. 59, 5151 (1973).

[39] E. Helfand and G.H. Fredrickson, Phys. Rev. Lett. 62, 2468 (1989).

[40] There is a freedom of scale change $m \rightarrow \lambda m$ and we may set $B=1$ without loss of generality. After this scale change $\delta \rho / \bar{\rho}=-\lambda \delta m-e_{1}$ determines the density deviation.

[41] C. Sagui, A. M. Somoza and R.C. Desai, Phys. Rev. E 50, 4865 (1994).

[42] J.W. Cahn, Acta Metall. 5, 160 (1957).

[43] A. Onuki and H. Nishimori, Phys. Rev. B 43, 13649 (1991); A. Onuki and A. Furukawa, Phys. Rev. Lett. 86, 452 (2001).

[44] D. Orlikowski, C. Sagui, A. M. Somoza and C. Roland, Phys. Rev. B 59, 8646 (1999); ibid. 62, 3160 (2000). 